\title{
Estratificação de um povoamento de eucalipto por interpoladores geoestatísticos e sensoriamento remoto
}

\author{
Aliny Aparecida dos Reis(1), José Márcio de Mello(1), Marcel Régis Raimundo(1), \\ Fausto Weimar Acerbi Júnior ${ }^{(1)}$, Marcelo Silva de Oliveira ${ }^{(2)}$ e Juliana Maria Ferreira de Souza Diniz ${ }^{(1)}$ \\ (1)Universidade Federal de Lavras (Ufla), Departamento de Ciências Florestais, Caixa Postal 3037, CEP 37200-000 Lavras, MG, \\ Brasil. E-mail: alinyreis@hotmail.com, josemarcio@dcf.ufla.br, marcelufla@gmail.com, fausto@dcf.ufla.br, juju-dinizsvm@hotmail.com \\ (2)Ufla, Departamento de Ciências Exatas, Caixa Postal 3037, CEP 37200-000 Lavras, MG, Brasil. E-mail: marcelo.oliveira@dex.ufla.br
}

\begin{abstract}
Resumo - O objetivo deste trabalho foi avaliar a utilização de interpoladores geoestatísticos e imagens de sensoriamento remoto, para a estratificação de povoamentos de Eucalyptus sp. em idade de corte. As estimativas da amostragem casual estratificada foram comparadas às estimativas da amostragem sistemática quanto à variável volume de madeira. A área de estudo correspondeu a um povoamento clonal de Eucalyptus sp. no Município de Lagoa Grande, MG. O inventário florestal foi realizado em 2011, em quatro situações de amostragem e diferentes números de parcelas. A estratificação da área foi feita por meio de krigagem da área basal (variável principal), da reflectância na banda TM5 (variável auxiliar) obtida em uma imagem Landsat 5 TM e com base na cokrigagem dessas variáveis. A área basal e a reflectância na banda TM5 apresentaram estrutura de dependência espacial. A estratificação reduziu o erro de amostragem em até $40 \%$, em comparação ao erro da amostragem sistemática. As imagens de sensoriamento remoto e os interpoladores geoestatísticos krigagem e cokrigagem são eficientes na definição dos estratos em povoamentos de Eucalyptus sp., em decorrência da maior precisão dos estimadores da amostragem casual estratificada, que permitem estimativas volumétricas mais precisas do inventário florestal do que os da amostragem sistemática.
\end{abstract}

Termos para indexação: Eucalyptus, cokrigagem, imagens, inventário florestal, krigagem.

\section{Stratification of an eucalyptus plantation through geostatistical interpolators and remote sensing}

\begin{abstract}
The objective of this work was to evaluate the use of geostatistical interpolators and remote sensing images for the stratification of Eucalyptus sp. stands, at harvesting age. Estimates by stratified random sampling and systematic sampling were compared for the timber volume variable. The study area was a clonal plantation of Eucalyptus sp. in the municipality of Lagoa Grande, in the state of Minas Gerais, Brazil. The forest inventory was performed in 2011, considering four sampling situations and different plot numbers. The area stratification was carried out based on kriging of the basal area (target variable), on the reflectance values in the TM5 band (auxiliary variable) extracted from a Landsat 5 TM image, and on the cokriging of these variables. Basal area and reflectance in the TM5 band showed a spatial dependence structure. Stratification reduced the sampling error up to $40 \%$, in comparison with that generated from systematic sampling. Remote sensing images and kriging and cokriging interpolators are efficient to define the strata in Eucalyptus sp. stands, as a consequence of the higher precision of the stratified random sampling estimators, which allow more precise volume estimates of the forest inventory than those of systematic sampling.
\end{abstract}

Index terms: Eucalyptus, cokriging, images, forest inventory, kriging.

\section{Introdução}

O inventário florestal contínuo (IFC) é o sistema de amostragem mais utilizado no Brasil para acompanhar o crescimento da floresta. O IFC consiste de uma rede de parcelas permanentes, monitoradas periodicamente, com o objetivo de prover bases inferenciais adequadas para a gestão do recurso florestal, levando-se em consideração as mudanças espaço-temporais ocorridas nas florestas (Tomppo et al., 2009; Westfall, 2016).

Durante o ciclo da floresta, a manutenção e o processamento das parcelas do IFC têm um custo significativo no processo de produção florestal. Além disso, as informações obtidas com o processamento do IFC não são consideradas suficientes, por parte dos gestores empresariais, para gerar estimativas seguras 
sobre o volume e o erro do inventário por projeto e por talhão, na idade de corte (Mello et al., 2006); assim, há necessidade de se efetuar o inventário pré-corte (IPC).

O inventário pré-corte é realizado a partir de parcelas temporárias adicionais, lançadas com maior intensidade amostral do que o IFC, naqueles talhões definidos para o corte, de acordo com o plano de suprimento das empresas florestais. Assim, são obtidas estimativas mais precisas, porém, a um custo mais elevado (Mello et al., 2009; Mengesha et al., 2014).

A utilização de interpoladores geoestatísticos, para a estratificação dos povoamentos florestais, surge como alternativa para a obtenção de maior precisão do IFC (Kanegae Júnior et al., 2006; Guedes et al., 2012; Roberge et al., 2016), para eliminar a necessidade da realização do inventário pré-corte.

Tradicionalmente, a estratificação de florestas plantadas é realizada com base em informações cadastrais, como a idade, a espécie, o espaçamento e o regime de manejo. Contudo, essas informações não levam em consideração a distribuição espacial dos diferentes estratos formados e possíveis relações entre estratos vizinhos. Assim, a utilização de interpoladores geoestatísticos, como a krigagem ordinária e a cokrigagem ordinária, oferece uma alternativa potencialmente eficiente para a busca de informações complementares que melhor caracterizem os povoamentos florestais, já que consideram explicitamente a informação espacial (Meng et al., 2009; Palmer et al., 2009; Akhavan \& Kia-Daliri, 2010; Viana et al., 2012; Singh \& Das, 2014), o que auxilia o processo de estratificação.

A utilização de informações obtidas em imagens de sensoriamento remoto, juntamente com os interpoladores geoestatísticos, no processo de estratificação, permite uma melhor caracterização dos povoamentos florestais, em razão da correlação entre a reflectância dos dosséis florestais e as variáveis dendrométricas dos povoamentos ( $\mathrm{Lu}$ et al., 2004; Meng et al., 2009; Berra et al., 2012; Almeida et al., 2014). Silva et al. (2014) conseguiram uma redução de aproximadamente $44 \%$ do erro da amostragem casual estratificada, em comparação à amostragem sistemática, ao utilizar uma imagem Landsat $5 \mathrm{TM}$ como base para a estratificação.

O objetivo deste trabalho foi avaliar a utilização de interpoladores geoestatísticos e imagens de sensoriamento remoto, para a estratificação de povoamentos de Eucalyptus sp. em idade de corte.

\section{Material e Métodos}

A área de trabalho correspondeu a um povoamento clonal de Eucalyptus sp., no Município de Lagoa Grande, no noroeste do Estado de Minas Gerais, entre $17^{\circ} 43^{\prime} 00^{\prime \prime} \mathrm{S}-17^{\circ} 44^{\prime} 00^{\prime \prime} \mathrm{S}$ e $46^{\circ} 32^{\prime} 00^{\prime \prime} \mathrm{W}-46^{\circ} 33^{\prime} 00^{\prime \prime} \mathrm{W}$, à altitude de $560 \mathrm{~m}$. O clima da região é tropical úmido e seco de savana, tipo Aw de acordo com a classificação de Köppen-Geiger, com meses mais secos durante o inverno. A precipitação anual média é de $1.430 \mathrm{~mm}$, e as precipitações médias dos períodos seco e úmido são de 8 e $257 \mathrm{~mm}$, respectivamente.

Este trabalho foi desenvolvido em um conjunto de 20 talhões, plantados entre 01/04/2004 e 29/05/2004,

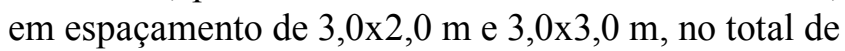
de 362,2 hectares. Os dados do inventário são oriundos de informações obtidas a partir de parcelas de $201,1 \mathrm{~m}^{2}$ (IPC) e $400 \mathrm{~m}^{2}$ (IFC), onde se realizou o inventário florestal no ano de 2011, correspondente à idade de corte do povoamento florestal.

O procedimento de amostragem adotado no inventário florestal foi o sistemático. Consideraramse quatro situações de amostragem, com diferentes números de parcelas (NP): NP1, uma parcela circular de $400 \mathrm{~m}^{2}$, a cada 10 ha de floresta (35 parcelas), correspondente à amostragem adotada no inventário florestal contínuo (IFC); NP2, uma parcela circular de $201,1 \mathrm{~m}^{2}$, a cada 5 ha de floresta (72 parcelas); NP3, uma parcela circular de $201,1 \mathrm{~m}^{2}$, a cada 3 ha de floresta (120 parcelas); e NP4, uma parcela circular de $201,1 \mathrm{~m}^{2}$, a cada 1,6 ha de floresta (222 parcelas). As situações NP2, NP3 e NP4 correspondem ao número de parcelas por hectare, comumente adotado no inventário pré-corte (IPC) pelas empresas florestais (Mello et al., 2009), e são superiores ao número de parcelas adotado no IFC.

Em todas as parcelas, mediu-se uma circunferência a 1,30 m do solo (CAP) de todos os fustes, a altura total das quinze primeiras árvores com fustes normais (sem bifurcação ou qualquer outro defeito) e a altura das árvores dominantes, caso estas não estivessem entre as quinze alturas já medidas da parcela. As parcelas foram sistematizadas sobre os shapefiles georreferenciados da área de estudo e localizadas em campo com GPS. A partir das informações coletadas das parcelas, foram 
obtidas as estimativas de área basal $\left(\mathrm{m}^{2} \mathrm{ha}^{-1}\right)$, altura dominante $(\mathrm{m})$ e volume $\left(\mathrm{m}^{3} \mathrm{ha}^{-1}\right)$.

Como variável secundária ou auxiliar, utilizaram-se os valores de reflectância, na região espectral do infravermelho médio, correspondente à banda TM5 de uma imagem do satélite Landsat 5 TM. A imagem Landsat 5 TM foi obtida do banco de dados da agência USGS (United States Geological Survey), já com as correções geométricas e radiométricas, na data de passagem 27/03/2011, correspondente à coleta dos dados em campo pelo inventário florestal, na órbita 220 ponto 072 , e resolução espacial de $30 \mathrm{~m}$. A USGS usa o aplicativo Landsat Ecosystem Disturbance Adaptive Processing System (Ledaps), que calcula a reflectância da superfície pela compensação dos efeitos de absorção e de espalhamento atmosféricos, gerando, deste modo, a correta reflectância do topo da atmosfera e da superfície (Feng et al., 2013).

A amostragem da variável auxiliar foi sistemática e formou uma malha amostral. A malha completa foi construída pela justaposição de duas malhas amostrais parciais, uma para as parcelas do inventário florestal, e outra com pontos na imagem correspondente à área de estudo, para a coleta dos valores de reflectância na banda TM5. A malha com as parcelas do inventário florestal foi denominada "parcelas", e a malha com pontos da imagem obtida por sensoriamento remoto foi denominada "conjunto amostral". A justaposição destas duas malhas foi feita de modo que cada conjunto amostral apresentasse alguns pontos coincidentes com as parcelas do inventário florestal. Os conjuntos amostrais CA1, CA2, CA3 e CA4 têm, respectivamente, $386,423,471$ e 573 pontos e apresentam 35, 72, 120 e 222 pontos coincidentes com as parcelas do inventário florestal, respectivamente. O modelo matemático adotado para a disposição das malhas foi o estabelecimento de uma densidade amostral pré-especificada - nos valores implícitos nas malhas de parcelas NP1, NP2, NP3 e NP4, e nas malhas de pontos dos conjuntos amostrais CA1, CA2, CA3 e CA4 -, aproximadamente constante, para cada pré-especificação, em toda a área de estudo.

Para a determinação do coeficiente de correlação linear de Pearson (r), entre os valores de área basal e os valores de reflectância na banda TM5, utilizaram-se os pontos coincidentes entre as parcelas do inventário florestal e os conjuntos amostrais.
As análises geoestatísticas univariadas e bivariadas foram realizadas, tendo-se considerado a pressuposição de estacionaridade da hipótese intrínseca (Journel \& Huijbregts, 1978), por meio de ajuste de funções teóricas aos modelos de semivariogramas e semivariogramas cruzados experimentais, conforme as equações a seguir:

$$
\begin{aligned}
& \gamma(\mathrm{h})=\frac{1}{2 \mathrm{~N}(\mathrm{~h})} \sum_{\mathrm{i}=1}^{\mathrm{N}(\mathrm{h})}\left[\mathrm{Z}\left(\mathrm{x}_{\mathrm{i}}\right)-\mathrm{Z}\left(\mathrm{x}_{\mathrm{i}}+\mathrm{h}\right)\right]^{2}, \\
& \gamma_{12}(\mathrm{~h})=\frac{1}{2 \mathrm{~N}(\mathrm{~h})}\left\{\sum_{\mathrm{i}=1}^{\mathrm{N}(\mathrm{h})}\left[\mathrm{Z}_{1}\left(\mathrm{x}_{1 \mathrm{i}}\right)-\mathrm{Z}_{1}\left(\mathrm{x}_{1 \mathrm{i}}+\mathrm{h}\right)\right]\left[\mathrm{Z}_{2}\left(\mathrm{x}_{2 \mathrm{i}}\right)-\mathrm{Z}_{2}\left(\mathrm{x}_{2 \mathrm{i}}+\mathrm{h}\right)\right]\right\},
\end{aligned}
$$

em que: $\mathrm{N}(\mathrm{h})$ é o número de pares experimentais de observações $Z\left(x_{i}\right)$ e $Z\left(x_{i}+h\right)$, separados por um vetor de distâncias $h$; $x_{i}$ é a posição espacial da variável $Z$; $Z_{1}$ e $Z_{2}$ são variáveis correlacionadas espacialmente.

Os modelos esférico, exponencial e gaussiano (Journel \& Huijbregts, 1978) foram ajustados pelo método dos mínimos quadrados ponderados. A avaliação do desempenho de cada modelo e a seleção dos melhores modelos foram feitas segundo o critério de validação cruzada (Vieira, 2000), pelo qual calcula-se o erro médio reduzido (EMR) e o desvio-padrão do erro médio reduzido $\left(S_{E R}\right)$, conforme abaixo:

$$
\begin{aligned}
& \mathrm{EMR}=\frac{1}{\mathrm{n}} \sum_{\mathrm{i}=1}^{\mathrm{n}} \frac{\mathrm{Z}\left(\mathrm{x}_{\mathrm{i} 0}\right)-\hat{\mathrm{Z}}\left(\mathrm{x}_{\mathrm{i} 0}\right)}{\sigma\left(\mathrm{x}_{\mathrm{i} 0}\right)}, \\
& \mathrm{S}_{\mathrm{ER}}=\sqrt{\frac{1}{\mathrm{n}} \sum_{\mathrm{i}=1}^{\mathrm{n}}\left(\frac{\mathrm{Z}\left(\mathrm{x}_{\mathrm{i} 0}-\hat{\mathrm{Z}}\left(\mathrm{x}_{\mathrm{i} 0}\right)\right.}{\sigma\left(\mathrm{x}_{\mathrm{i} 0}\right)}\right)^{2}},
\end{aligned}
$$

em que: $\widehat{Z}\left(\mathrm{x}_{\mathrm{i} 0}\right)$ é o valor estimado no ponto $\mathrm{i} 0$; $\mathrm{Z}\left(\mathrm{x}_{\mathrm{i} 0}\right)$ é o valor estimado para o ponto i0; e $\sigma\left(\mathrm{x}_{\mathrm{i} 0}\right)$ é o desvio-padrão da krigagem no ponto i0.

No ajuste dos modelos teóricos aos semivariogramas experimentais, determinaram-se os parâmetros efeito pepita $\left(\tau^{2}\right)$, patamar $\left(\sigma^{2}\right)$ e alcance $(\phi)$. Para a análise do grau de dependência espacial (DE), utilizaramse a relação $\tau^{2} /\left(\tau^{2}+\sigma^{2}\right)$ e os intervalos propostos por Cambardella et al. (1994), que consideram as seguintes dependências espaciais: forte, $\mathrm{DE}<25 \%$; moderada, $25 \%<\mathrm{DE}<75 \%$; e fraca, $\mathrm{DE}>75 \%$.

Em locais não amostrados, realizaram-se a partir da krigagem ordinária as estimativas dos valores de área basal nos números de parcelas - NP1, NP2, NP3 e NP4, os de reflectância na banda TM5 - nos conjuntos amostrais CA1, CA2, CA3 e CA4 - e a confecção dos mapas de distribuição espacial dessas variáveis. A krigagem ordinária é um interpolador

Pesq. agropec. bras., Brasília, v.51, n.10, p.1751-1761, out. 2016 DOI: $10.1590 / \mathrm{S} 0100-204 X 2016001000007$ 
geoestatístico para estimar, sem viés e com erro mínimo, os valores de pontos não amostrados, com base nos pontos amostrados, levando-se em consideração a estrutura de dependência espacial dos dados (Journel \& Huijbregts, 1978). O estimador de krigagem ordinária é dado por:

$$
\widehat{Z}_{\mathrm{x} 0}=\sum_{\mathrm{i}=1}^{\mathrm{n}} \lambda_{\mathrm{i}} \mathrm{Z}_{\mathrm{xi}},
$$

em que: $\widehat{Z}_{\mathrm{x} 0}$ é a estimativa do valor na posição $\mathrm{x}_{0}$ (média ponderada dos dados); n é o número de pontos amostrais vizinhos, utilizados para a predição do valor não amostrado (vizinhança da krigagem); e $\lambda_{i}$ é o i-ésimo peso, atribuído a cada i-ésima observação da variável de interesse, na posição $\mathrm{x}, \mathrm{Z}_{\mathrm{xi}}$, em que estes pesos são calculados pelo sistema de krigagem definido pelo semivariograma teórico ajustado (Journel \& Huijbregts, 1978).

Para estimar a área basal em função da reflectância na banda TM5, em cada número de parcela (NP) e conjunto amostral (CA), utilizou-se a extensão bivariada da krigagem ordinária, conhecida como cokrigagem ordinária (Isaaks \& Srivastava, 1989). Esse método baseia-se nos parâmetros expressos por um semivariograma cruzado entre duas variáveis, para a estimativa de novos valores em locais não amostrados (Yamamoto \& Landim, 2013). A estimativa de uma variavel $\widehat{Z}_{x 0}$ para qualquer local $x_{0}$ deve ser uma combinação linear de $Z_{1}$ e $Z_{2}$, conforme abaixo:

$$
\widehat{Z}_{\mathrm{x} 0}=\sum_{\mathrm{i}=1}^{\mathrm{n}_{1}} \lambda_{1 \mathrm{i}} \mathrm{Z}_{1}\left(\mathrm{x}_{1 \mathrm{i}}\right)+\sum_{\mathrm{j}=1}^{\mathrm{n}_{2}} \lambda_{2 \mathrm{j}} \mathrm{Z}_{2}\left(\mathrm{x}_{2 \mathrm{j}}\right)
$$

em que: $\mathrm{n}_{1}$ e $\mathrm{n}_{2}$ são os números de vizinhos de $Z_{1}$ e $Z_{2}$, respectivamente; $\lambda_{1}$ e $\lambda_{2}$ são os pesos associados a cada valor de $Z_{1}$ e $Z_{2}$, que são distribuídos de acordo com a dependência espacial de cada uma das variáveis entre si e com a correlação cruzada entre elas. Assim como na krigagem, o estimador da cokrigagem é ótimo, além de apresentar variância mínima e não tendenciosidade (Yamamoto \& Landim, 2013).

Nas estratificações obtidas pela krigagem e cokrigagem das diferentes variáveis estratificadoras comparadas (Tabela 1), foram formados três estratos. O número de estratos foi definido em razão da exigência da localização do mínimo de três parcelas em cada estrato, para possibilitar a estimativa da variância no processamento do inventário com os estimadores da amostragem casual estratificada. Os estratos são definidos como sub-regiões, dentro da área total, que apresentam valores da variável estratificadora mais homogêneos do que os da população heterogênea.

Para avaliar o desempenho dos estratificadores, realizou-se o processamento do inventário florestal quanto à variável de interesse volume de madeira $\left(\mathrm{m}^{3} \mathrm{ha}^{-1}\right)$ por parcela. Consideraram-se as seguintes situações para a estimativa volumétrica: adoção da área em estudo como um todo, sem a delimitação dos estratos, com processamento do inventário realizado com os estimadores da amostragem sistemática (AS); considerando-se as estratificações geradas pela krigagem e cokrigagem, com o processamento do inventário efetuado pelos estimadores da amostragem casual estratificada (ACE), listados a seguir:

Tabela 1. Estratificadores correspondentes às interpolações geoestatísticas da krigagem ordinária e da cokrigagem ordinária, quanto aos valores de área basal, nas parcelas, e aos valores de reflectância, na banda TM5, para os diferentes conjuntos amostrais, em povoamento clonal de Eucalyptus sp., em Lagoa Grande, MG.

\begin{tabular}{lc}
\hline Código & Estratificador \\
\hline KoG-NP1 & Krigagem ordinária da área basal no NP1 \\
KoTM5-CA1 & Krigagem ordinária da reflectância na banda TM5, no CA1 \\
CoK-NP1-CA1 & Cokrigagem ordinária da área basal, no NP1, e da reflectância na banda TM5, no CA1 \\
KoG-NP2 & Krigagem ordinária da área basal no NP2 \\
KoTM5-CA2 & Krigagem ordinária da reflectância na banda TM5, no CA2 \\
CoK-NP2-CA2 & Cokrigagem ordinária da área basal, no NP2, e da reflectância na banda TM5, no CA2 \\
KoG-NP3 & Krigagem ordinária da área basal no NP3 \\
KoTM5-CA3 & Krigagem ordinária da reflectância na banda TM5, no CA3 \\
CoK-NP3-CA3 & Cokrigagem ordinária da área basal, no NP3, e da reflectância na banda TM5, no CA3 \\
KoG-NP4 & Krigagem ordinária da área basal no NP4 \\
KoTM5-CA4 & Krigagem ordinária da reflectância na banda TM5, no CA4 \\
CoK-NP4-CA4 & Cokrigagem ordinária da área basal, no NP4, e da reflectância na banda TM5, no CA4 \\
\hline
\end{tabular}

NP, número de parcelas; CA, conjunto amostral; Ko, krigagem ordinária; CoK, cokrigagem ordinária. 


$$
\begin{aligned}
& \bar{Y}_{s t r}=\sum_{j=1}^{n} \frac{N_{j}}{N} \times \bar{Y}_{j}, S_{\bar{Y}_{s t r}}^{2}=\sum_{j=1}^{n}\left(\frac{N_{j}}{N}\right)^{2} \times \frac{S_{j}^{2}}{n_{j}} \times\left(1-\frac{n_{j}}{N_{j}}\right), \\
& E=\sqrt{S_{\bar{S}_{s t r}}^{2}} \times t,
\end{aligned}
$$

em que: $\overline{\mathrm{Y}}_{\text {str }}$ corresponde à média da população estratificada; $\overline{\mathrm{Y}}_{\mathrm{j}}$ é a média aritmética das parcelas amostradas no estrato j; n é o número de estratos; $\mathrm{N}_{\mathrm{j}}$ é o número de parcelas cabíveis no estrato $\mathrm{j}$; $\mathrm{N}$ é o número de parcelas cabíveis na população; $\mathrm{n}_{\mathrm{j}}$ é o número de parcelas amostradas no estrato $\mathrm{j} ; \mathrm{S}_{\mathrm{j}}{ }_{\mathrm{j}}$ é a variância das parcelas amostradas no estrato $j$; $S_{\bar{Y} \text { str }}^{2}$ $\mathrm{S}^{2}{ }_{\text {Ystr }}$ corresponde à variância da média da população estratificada; E corresponde ao erro de estimação da média imposto pela amostragem, ou, aqui, também denominado como erro de amostragem; e t é o valor tabelado da distribuição t de Student.

Após a estratificação, definiram-se as parcelas de cada estrato, e os processamentos da ACE foram comparados àqueles da AS. A formulação detalhada dos estimadores da AS e da ACE pode ser verificada em Batista et al. (2014). A comparação da estimativa volumétrica obtida a partir das estratificações foi realizada por meio do erro de estimação da média imposto pela amostragem (erro de amostragem) e da sobreposição dos intervalos de confiança, obtidos a partir da média e do erro de amostragem (Mello et al., 2006). A ocorrência de tal sobreposição indica que as estimativas de média a partir das diferentes estratificações são semelhantes.

A análise de sobreposição dos mapas das estratificações foi feita comintuito de identificar as áreas de coincidência entre as estratificações. Os programas R (R Core Team, 2014) e seu pacote geoR (Ribeiro Júnior \& Diggle, 2001) e GS+ versão 10 (Robertson, 1998) foram utilizados para o ajuste e seleção de modelos aos semivariogramas e aos semivariogramas cruzados experimentais. As interpolações foram realizadas no ArcGis versão 10.1 (Environmental Systems Research Institute, Redlands, CA, EUA), por meio da extensão Geostatystical Analyst, assim como a confecção dos mapas.

\section{Resultados e Discussão}

O teste de correlação de Pearson entre a variável principal (área basal) e a variável auxiliar (reflectância na banda TM5) resultou em correlações inversas e significativas entre essas duas variáveis. A banda TM5 é a banda espectral que está mais fortemente correlacionada às características biométricas dos povoamentos florestais, pois é independente das diferenças entre os ambientes biofísicos (Lu etal., 2004). A existência de correlação entre a variável principal e a variável auxiliar é indispensável para a eficiência da cokrigagem (Aertsen et al., 2012). No entanto, mesmo não existindo evidências de altos coeficientes de correlação linear simples entre a variável principal e a variável auxiliar, esse fato não invalida a hipótese da existência de uma estrutura de dependência espacial entre a combinação dessas variáveis, desde que a correlação não seja nula (Kitamura et al., 2007).

Todas as variáveis apresentaram estrutura de dependência espacial definida (Figura 1 e Tabela 2) e grau de dependência espacial forte, com exceção da KoG-NP4, que apresentou grau de dependência espacial moderado, segundo a classificação proposta por Cambardella et al. (1994). A reflectância na banda TM5 (variável auxiliar) apresentou melhor estrutura de dependência espacial do que a área basal (variável principal), em todos os NPs. Este fato é benéfico quando se deseja utilizar uma variável como auxiliar na cokrigagem. Uma das situações em que a utilização da cokrigagem produz resultados satisfatórios ocorre quando a variável auxiliar apresenta melhor estrutura de dependência espacial do que a variável principal (Yamamoto \& Landim, 2013).

Os estratos gerados pela krigagem da área basal e da reflectância na banda TM5 (Figura 2) e pela cokrigagem dessas variáveis (Figura 3) apresentaram grande semelhança entre a localização espacial dos estratos. As estratificações geradas pela reflectância na banda TM5 conseguiram discretizar melhor as classes de produtividade do que as estratificações oriundas das observações em campo (área basal). Porém, essas estratificações apresentaram menor percentagem de áreas coincidentes com as estratificações obtidas a partir da área basal (krigagem e cokrigagem). As estratificações obtidas a partir da cokrigagem ordinária conseguiram captar melhor a distribuição espacial dos estratos, em razão do maior poder de discriminação e do elevado número de amostras da variável auxiliar reflectância na banda TM5. Esse fato pode trazer ganhos substanciais ao processamento do inventário florestal, pois, a delimitação precisa e o melhor detalhamento das áreas dos estratos são 
de fundamental importância para a utilização dos estimadores da ACE.

A diferença do número de parcelas (NP) de cada amostragem da área basal não afetou significativamente a localização espacial dos estratos, uma vez que as estratificações apresentam altas correlações entre si. Assim, a amostragem do IFC proporcionou estratos semelhantes em relação àquelas situações com maior número de parcelas. Logo, o uso das informações do IFC, com dados auxiliares obtidos em imagens de sensoriamento remoto, constitui uma estratégia interessante para efetuar a estratificação em plantios de eucalipto em idade de corte, pois, essas informações apresentam potencial para serem utilizadas como substitutos do IPC.

O erro de amostragem do inventário variou de 3,11 a $10,77 \%$ para a AS, nos diferentes NPs, e variou de 2,31 a $7,87 \%$, para as diferentes estratificações geradas
(Tabela 3). Os menores erros foram observados para as estratificações KoG-NP4, KoTM5-CA4 e CoK-NP4-CA4, que consideraram um grande número de parcelas no processamento do inventário. Esse fato pode acarretar aumentos de custos operacionais da amostragem, uma vez que são fortemente influenciados pelo tempo gasto no processo de demarcação e medição das unidades amostrais em campo (Guedes et al., 2012).

A utilização de dados obtidos de imagens de sensoriamento remoto, para a estratificação dos povoamentos de Eucalyptus sp., proporcionou redução do erro de amostragem do inventário, quando as imagens foram utilizadas individualmente. No entanto, quando a reflectância na banda TM5 foi utilizada como variável auxiliar na cokrigagem, nos números de parcelas NP2, NP3 e NP4, o erro de amostragem obtido a partir da cokrigagem foi ligeiramente
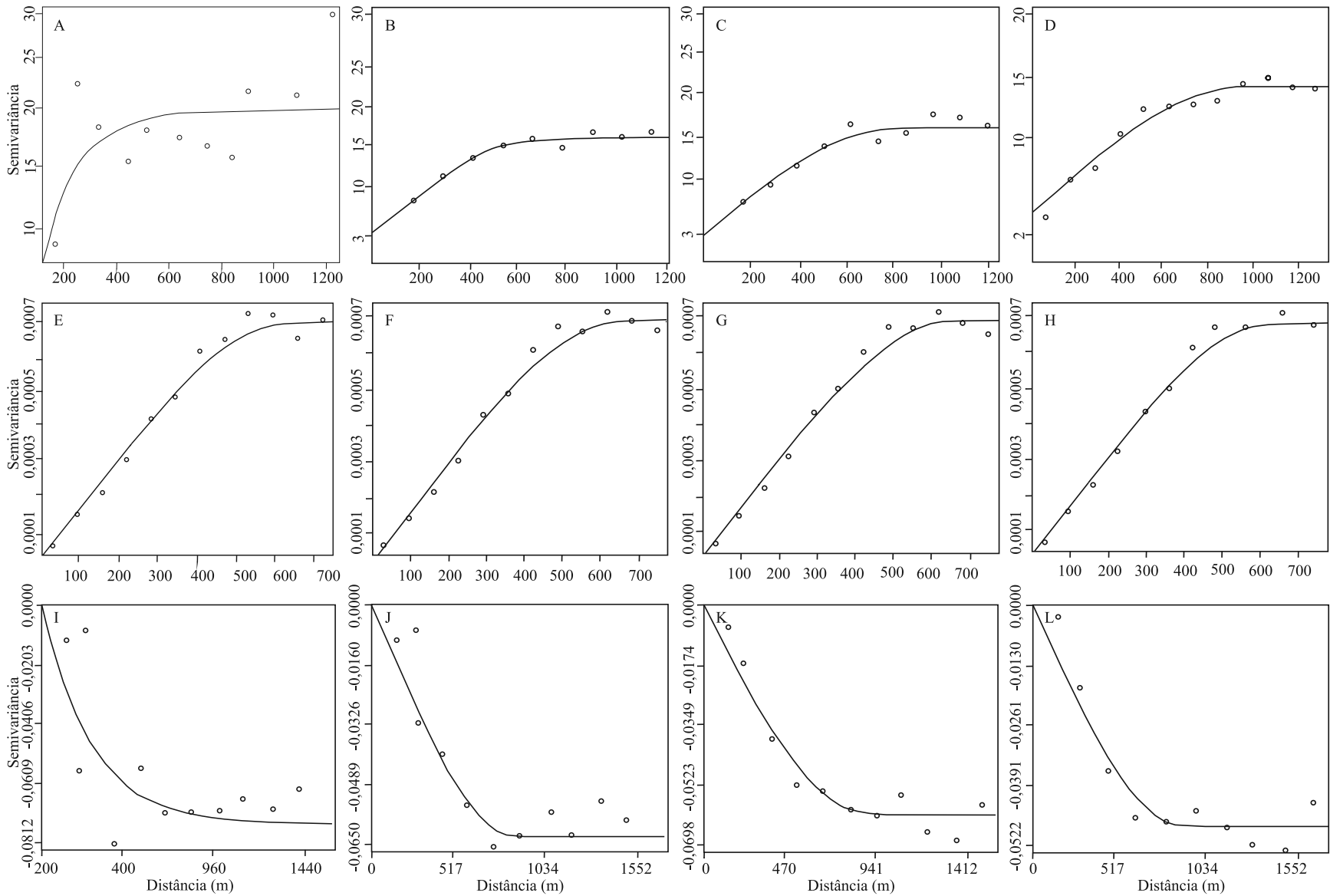

Figura 1. Semivariogramas experimentais para os dados de área basal, nos números de parcelas NP1 (A), NP2 (B), NP3 (C), NP4 (D); de reflectância na banda TM5, nos conjuntos amostrais CA1 (E), CA2 (F), CA3 (G) e CA4 (H); e semivariogramas cruzados dessas variáveis nos NP1-CA1 (I), NP2-CA2 (J), NP3-CA3 (K), NP4-CA4 (L), em povoamento clonal de Eucalyptus sp., Lagoa Grande, MG. 
superior ao erro de amostragem obtido apenas com a estratificação da área basal pela krigagem, nesses NPs. Esse fato ocorreu em razão das diferenças de número de parcelas utilizadas pela cokrigagem em cada amostragem. Nas situações em que a variável principal foi pouco amostrada, a incorporação da variável auxiliar acarretou a melhoria das estimativas da variável principal e, consequentemente, menores erros de amostragem. Nas situações em que a variável principal foi amostrada em alta intensidade, o efeito da variável auxiliar foi pouco pronunciado, e sua incorporação não trouxe ganhos para o processo de estimativa da variável principal.

A redução do erro de amostragem do processamento do inventário, pormeio do estratificador CoK-NP1-CA1, em comparação à AS no NP1, foi de aproximadamente $40 \%$. Nos números de parcelas NP2, NP3 e NP4, houve redução de 37,34, 33,69 e 25,72\%, respectivamente, quanto ao erro da $\mathrm{ACE}$, obtida por meio da krigagem da área basal, em comparação à AS. Os intervalos de confiança são semelhantes entre si, o que evidencia que a estratificação com base nos interpoladores geoestatísticos produz resultados satisfatórios quanto à redução do erro de amostragem do inventário.

Aplicando-se a cokrigagem aos valores de área basal no NP1 e reflectância na banda TM5 no CA1 obtém-se, praticamente, o mesmo erro do processamento do inventário pela AS no NP2, em que foi medido o dobro de parcelas do que no NP1. Ao se utilizar a cokrigagem nos dados de área basal no
NP2 e reflectância na banda TM5 no CA2, obteve-se o mesmo erro do processamento do inventário pelos estimadores da AS no NP3; no NP2, foram medidas 72 parcelas e, no NP3, 120 parcelas. O mesmo raciocínio pode ser aplicado à comparação do erro de amostragem do processamento do inventário por meio dos estratificadores KoG-NP3 e AS-NP4. Esse fato implica diretamente a redução do número de parcelas necessário para se obter um determinado erro do inventário máximo, pré-estabelecido pelas empresas florestais.

Os resultados aqui observados indicam que a utilização de estratificadores geoestatísticos e imagens de sensoriamento remoto pode substituir o trabalho do inventário pré-corte, ou mesmo reduzir a intensidade amostral em que são realizados, uma vez que se consegue reduzir o erro de amostragem em até $40 \%$, por meio apenas da estratificação dos dados do IFC. Essa mesma redução de $40 \%$ é observada, ao se utilizar o dobro do número de parcelas (uma parcela a cada 5 ha) no IPC, em comparação ao IFC (uma parcela a cada $10 \mathrm{ha}$ ).

Os dados obtidos em imagens de sensoriamento remoto podem ser adquiridos em uma elevada intensidade amostral e a custos relativamente baixos e, caso não haja informações de campo, estes dados podem ser utilizados para pré-estratificações de povoamentos de Eucalyptus, desde que identificada previamente a variável que melhor se correlacione às características dendrométricas do povoamento.

Tabela 2. Parâmetros efeito pepita $\left(\tau^{2}\right)$, patamar $\left(\sigma^{2}\right)$ e alcance teórico $(\phi)$, e grau de dependência espacial (DE) para os modelos de função de semivariância, selecionados para cada uma das variáveis, em povoamento clonal de Eucalyptus sp., em Lagoa Grande, MG.

\begin{tabular}{|c|c|c|c|c|c|c|c|}
\hline Variável e amostragem & Modelo selecionado & $\tau^{2}$ & $\sigma^{2}$ & $\phi(\mathrm{m})$ & DE (\%) & EMR & $S_{E R}$ \\
\hline KoG-NP1 & Exponencial & 0 & 20,05 & 194 & 0 & 0,0142 & 0,981 \\
\hline KoTM5-CA1 & Esférico & 0 & $7,048 \times 10^{-4}$ & 673 & 0 & $-0,0028$ & 1,042 \\
\hline CoK-NP1-CA1 & Exponencial & $-0,0001$ & $-0,0752$ & 260 & 0,13 & 0,0107 & 1,017 \\
\hline KoG-NP2 & Esférico & 3,83 & 12,35 & 730 & 23,67 & 0,0041 & 0,956 \\
\hline KoTM5-CA2 & Esférico & $3,668 \times 10^{-5}$ & $6,912 \times 10^{-4}$ & 667 & 5,04 & 0,0031 & 1,037 \\
\hline CoK-NP2-CA2 & Esférico & $-0,0001$ & $-0,06250$ & 797 & 0,16 & $-0,0014$ & 0,989 \\
\hline KoG-NP3 & Esférico & 3,01 & 13,09 & 825 & 18,7 & 0,0003 & 0,998 \\
\hline KoTM5-CA3 & Esférico & 0 & $7,027 \times 10^{-4}$ & 669 & 0 & 0,0122 & 0,975 \\
\hline CoK-NP3-CA3 & Esférico & $-0,0001$ & $-0,0612$ & 783 & 0,16 & $-0,0011$ & 1,008 \\
\hline KoG-NP4 & Esférico & 3,89 & 9,99 & 998 & 28,03 & $-0,0019$ & 0,992 \\
\hline KoTM5-CA4 & Esférico & $4,782 \times 10^{-5}$ & $6,458 \times 10^{-4}$ & 661 & 6,90 & 0,0047 & 0,980 \\
\hline CoK-NP4-CA4 & Esférico & $-0,0001$ & $-0,0473$ & 805 & 0,21 & $-0,0009$ & 0,999 \\
\hline
\end{tabular}

Ko, krigagem ordinária; CoK, cokrigagem ordinária; G, área basal; TM5, reflectância na banda TM5; NP, número de parcelas; CA, conjunto amostral; EMR, erro médio reduzido; $\mathrm{S}_{\mathrm{ER}}$, desvio-padrão do erro médio reduzido. 

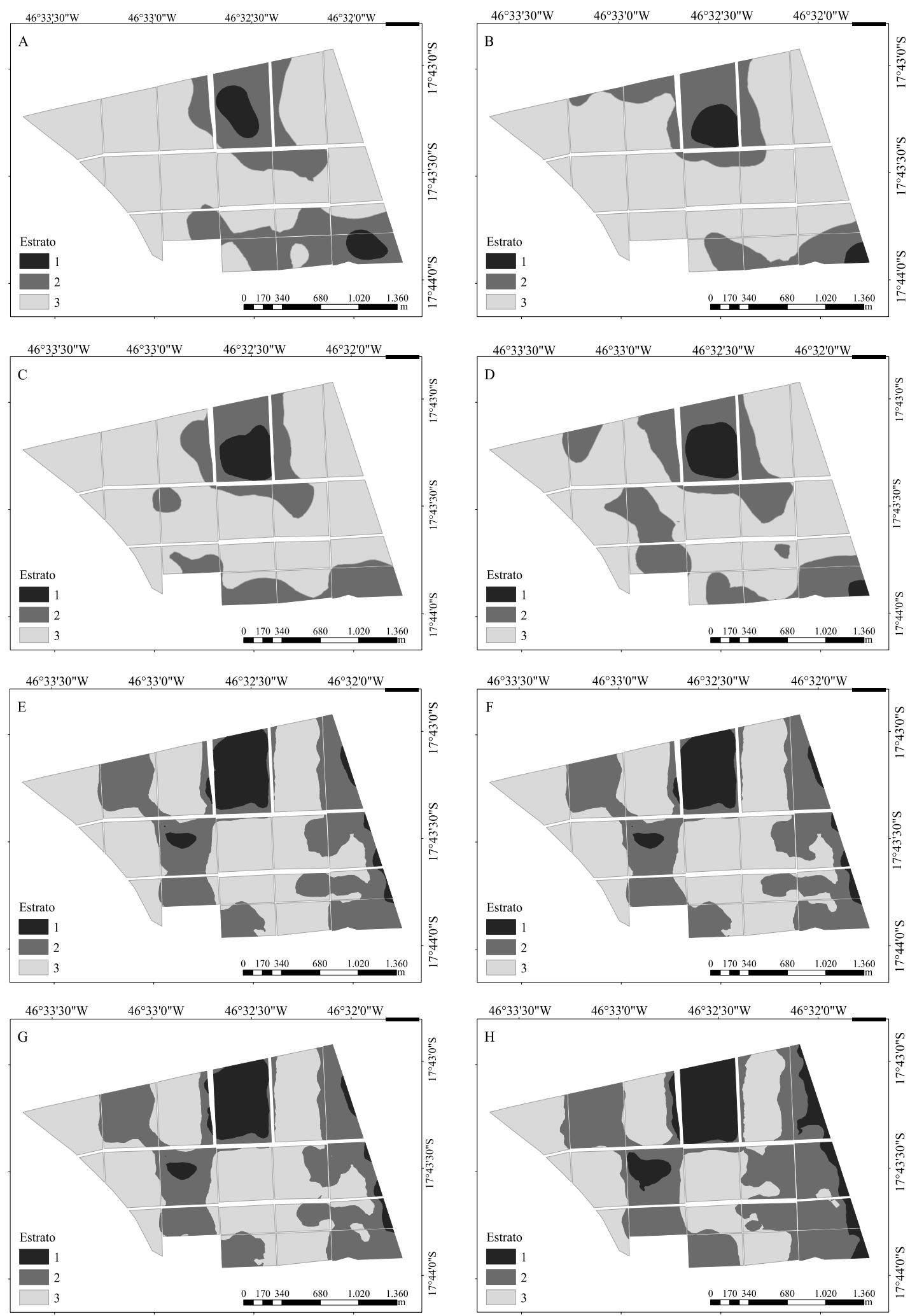

Figura 2. Estratificações geradas a partir da krigagem ordinária dos dados de área basal, nos números de parcelas NP1 (A), NP2 (B), NP3 (C), NP4 (D), e dos dados de reflectância na banda TM5, nos conjuntos amostrais CA1 (E), CA2 (F), CA3 (G) e CA4 (H), para o povoamento clonal de Eucalyptus sp., em Lagoa Grande, MG. 

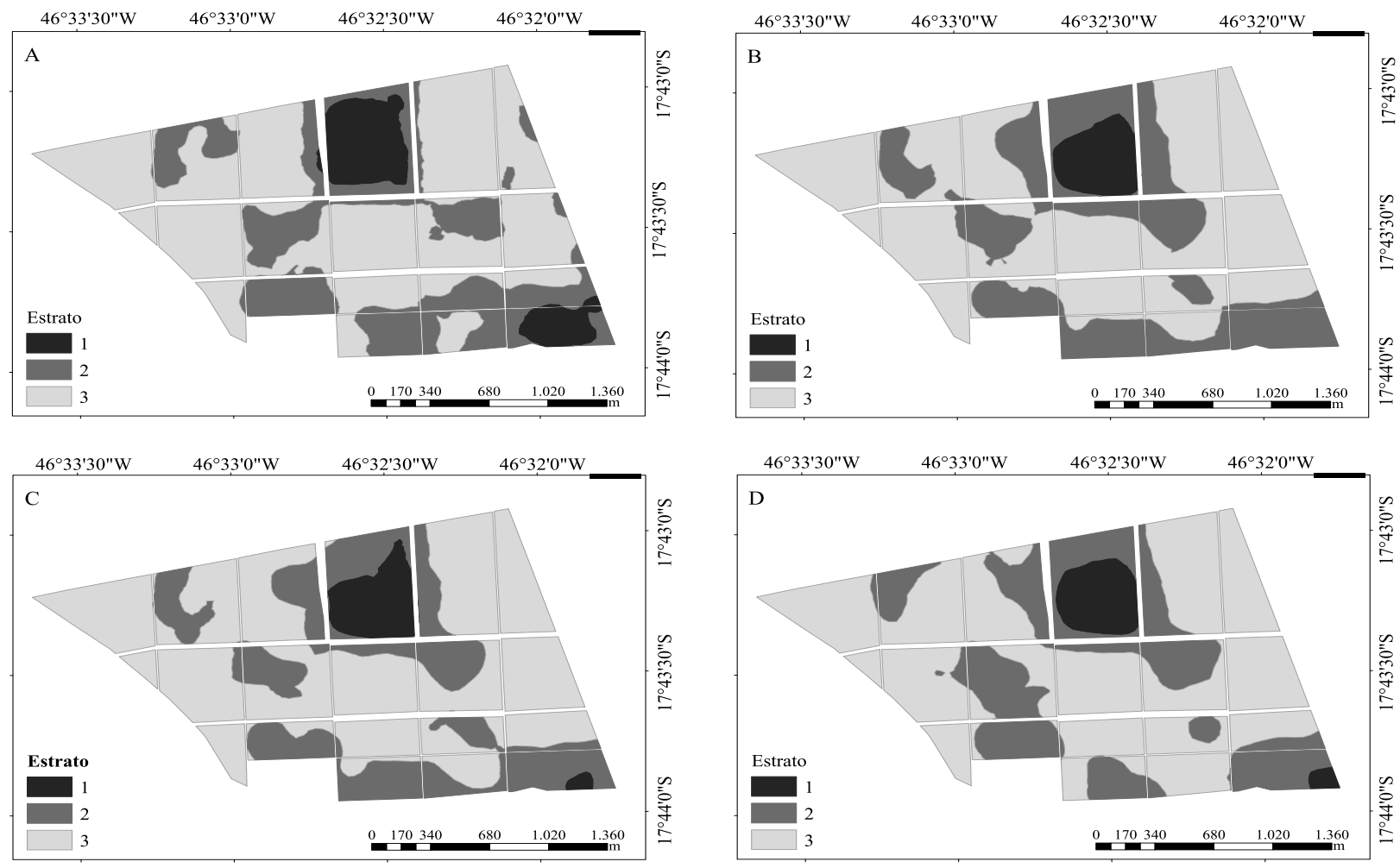

Figura 3. Estratificações geradas a partir da cokrigagem ordinária da área basal e da reflectância na banda TM5, nos números de parcela (NP): NP1 (A), NP2 (B), NP3 (C) e NP4 (D), para o povoamento clonal de Eucalyptus sp., em Lagoa Grande, MG.

Tabela 3. Volume médio, desvio-padrão, erro do inventário, erro de amostragem e intervalo de confiança da amostragem sistemática, e as diferentes amostragens casuais estratificadas, em povoamento clonal de Eucalyptus sp., em Lagoa Grande, MG.

\begin{tabular}{|c|c|c|c|c|c|}
\hline Estimadores & $\begin{array}{c}\text { Volume médio } \\
\left(\mathrm{m}^{3} \mathrm{ha}^{-1}\right)\end{array}$ & $\begin{array}{c}\text { Desvio-padrão } \\
\left(\mathrm{m}^{3} \mathrm{ha}^{-1}\right)\end{array}$ & $\begin{array}{c}\text { Erro do inventário } \\
\left(\mathrm{m}^{3} \mathrm{ha}^{-1}\right)\end{array}$ & $\begin{array}{c}\text { Erro de amostragem } \\
(\%)\end{array}$ & $\begin{array}{c}\text { Intervalo de confiança } \\
\left(\mathrm{m}^{3} \mathrm{ha}^{-1}\right)\end{array}$ \\
\hline AS-NP1 & 209,71 & 62,83 & 21,54 & 10,27 & $188,17-231,25$ \\
\hline KoG-NP1 & 211,63 & 41,69 & 15,98 & 7,55 & $195,65-227,61$ \\
\hline KoTM5-CA1 & 208,28 & 48,64 & 16,39 & 7,87 & $191,90-224,67$ \\
\hline CoK-NP1-CA1 & 211,39 & 36,98 & 12,91 & 6,11 & $198,48-224,30$ \\
\hline AS-NP2 & 215,10 & 56,61 & 13,25 & 6,16 & $201,85-228,35$ \\
\hline KoG-NP2 & 216,40 & 35,55 & 8,35 & 3,86 & $208,05-224,75$ \\
\hline KoTM5-CA2 & 214,56 & 43,88 & 10,24 & 4,77 & $204,32-224,81$ \\
\hline CoK-NP2-CA2 & 214,91 & 42,90 & 10,05 & 4,68 & $204,86-224,96$ \\
\hline AS-NP3 & 213,50 & 55,38 & 9,94 & 4,66 & $203,56-223,44$ \\
\hline KoG-NP3 & 212,43 & 36,11 & 6,56 & 3,09 & $205,87-219,00$ \\
\hline KoTM5-CA3 & 215,73 & 43,84 & 7,76 & 3,60 & $207,97-223,48$ \\
\hline CoK-NP3-CA3 & 213,27 & 37,99 & 6,85 & 3,21 & $206,43-220,12$ \\
\hline AS-NP4 & 217,41 & 51,68 & 6,75 & 3,11 & $210,65-224,16$ \\
\hline KoG-NP4 & 215,34 & 37,88 & 4,97 & 2,31 & $210,37-220,31$ \\
\hline KoTM5-CA4 & 215,97 & 46,97 & 6,37 & 2,95 & $209,60-222,34$ \\
\hline CoK-NP4-CA4 & 215,32 & 38,46 & 5,04 & 2,34 & $210,28-220,36$ \\
\hline
\end{tabular}

AS, amostragem sistemática; KoG-NP1, krigagem da área basal, no NP1; KoTM5-CA1, krigagem da reflectância na banda TM5, no CA1; CoK-NP1-CA1, cokrigagem da área basal, no NP1, e da reflectância na banda TM5, no CA1; KoG-NP2, krigagem da área basal, no NP2; KoTM5-CA2, krigagem da reflectância na banda TM5, no CA2; CoK-NP2-CA2, cokrigagem da área basal, no NP2, e da reflectância na banda TM5, no CA2; KoG-NP3, krigagem da área basal, no NP3; KoTM5-CA3, krigagem da reflectância na banda TM5, no CA3; CoK-NP3-CA3, cokrigagem da área basal, no NP3, e da reflectância na banda TM5, no CA3; KoG-NP4, krigagem da área basal, na NP4; KoTM5-CA4, krigagem da reflectância na banda TM5, no CA4; CoK-NP4-CA4, cokrigagem da área basal, na NP4, e da reflectância na banda TM5, no CA4. 
A utilização de imagens de sensoriamento remoto para a estratificação, antes do levantamento de campo, proporciona uma melhor distribuição espacial das parcelas e evita que ocorram estratos sem amostragem adequada ou amostragem de áreas impróprias ao manejo florestal (Silva et al., 2014).

Kanegae Júnior et al. (2006) utilizaram a amostragem de uma parcela, a cada 10 ha de floresta, e verificaram a redução de $66 \%$ da variabilidade do povoamento, ao efetuarem a estratificação com base na variável de interesse volume em povoamentos de eucalipto, por meio da krigagem ordinária como ferramenta de estratificação. Guedes et al. (2012) avaliaram a possibilidade de uso da estrutura de dependência espacial, para a definição de estratos com base no volume, em povoamentos florestais, por meio da amostragem de uma parcela a cada 10 ha de floresta, e observaram redução do erro de amostragem que variou de 47,0 a $68,4 \%$ da $\mathrm{ACE}$, em relação à ACS. Essa variação foi ocasionada pelo fato de o grau de dependência espacial ter variado de fraco a forte entre os projetos avaliados. Os resultados desses autores corroboram o potencial de utilização da estratificação para a redução do erro de amostragem e, consequentemente, a redução do número de parcelas utilizadas nos inventários pelas empresas florestais, sem perda de precisão das estimativas.

\section{Conclusões}

1. Os interpoladores geoestatísticos krigagem ordinária e cokrigagem ordinária são eficientes para a definição de estratos em povoamentos de Eucalyptus sp., pois os estimadores da amostragem casual estratificada permitem estimativas volumétricas mais precisas do inventário florestal do que os estimadores da amostragem sistemática.

2. As imagens de sensoriamento remoto são eficientes, quando utilizadas individualmente ou como auxiliares da cokrigagem, no processo de estratificação do inventário florestal.

\section{Referências}

AERTSEN, W.; KINT, V.; WILPERT, K. von; ZIRLEWAGEN, D.; MUYS, B.; ORSHOVEN, J. van. Comparison of location-based, attribute-based and hybrid regionalization techniques for mapping forest site productivity. Forestry, v.85, p.539-550, 2012. DOI: 10.1093 /forestry/cps 050 .
AKHAVAN, R.; KIA-DALIRI, H. Spatial variability and estimation of tree attributes in a plantation forest in the Caspian region of Iran using geostatistical analysis. Caspian Journal of Environmental Sciences, v.8, p.163-172, 2010.

ALMEIDA, A.Q. de; MELLO, A.A. de; DÓRIA NETO, A.L.; FERRAZ, R.C. Relações empíricas entre características dendrométricas da Caatinga brasileira e dados TM Landsat 5. Pesquisa Agropecuária Brasileira, v.49, p.306-315, 2014. DOI: 10.1590/S0100-204X2014000400009.

BATISTA, J.L.F.; COUTO, H.T.Z. do; SILVA FILHO, D.F. da. Quantificação de recursos florestais: árvores, arvoredos e florestas. São Paulo: Oficina de Textos, 2014. 384p.

BERRA, E.F.; BRANDELERO, C.; PEREIRA, R.S.; SEBEM, E.; GOERGEN, L.C. de G.; BENEDETTI, A.C.P.; LIPPERT, D.B. Estimativa do volume total de madeira em espécies de eucalipto a partir de imagens de satélite Landsat. Ciência Florestal, v.22, p.853-864, 2012. DOI: 10.5902/198050987566.

CAMBARDELLA, C.A.; MOORMAN, T.B.; NOVAK, J.M.; PARKIN, T.B.; KARLEN, D.L.; TURCO, R.F.; KONOPKA, A.E. Field-scale variability of soil properties in central Iowa soils. Soil Science Society of America Journal, v.58, p.1501-1511, 1994.

ENVIRONMENTAL SYSTEMS RESEARCH INSTITUTE ArcGIS Desktop: Release 10.1. Redlands, CA: ESRI, 2010.

FENG, M.; SEXTON, J.O.; HUANG, C.; MASEK, J.G.; VERMOTE, E.F.; GAO, F.; NARASIMHAN, R.; CHANNAN, S.; WOLFE, R.E.; TOWNSHEND, J.R. Global surface reflectance products from Landsat: assessment using coincident MODIS observations. Remote Sensing of Environment, v.134, p.276-293, 2013. DOI: 10.1016/j.rse.2013.02.031.

GUEDES, I.C. de L.; MELlO, J.M. de; MELlO, C.R. de; OLIVEIRA, A.D. de; SILVA, S.T. da; SCOLFORO, J.R.S. Técnicas geoestatísticas e interpoladores espaciais na estratificação de povoamentos de Eucalyptus sp. Ciência Florestal, v.22, p.541-550, 2012. DOI: 10.5902/198050986621.

ISAAKS, E.H.; SRIVASTAVA, R.M. Applied geostatistics. Oxford: Oxford University 1989. 561p.

JOURnEL, A.G.; HUIJBREGTS, C.J. Mining geostatistics. London: Academic Press, 1978. 600p.

KANEGAE JÚNIOR, H.; SCOLFORO, J.R.S.; MELLO, J.M. de; OLIVEIRA, A.D. de. Avaliação de interpoladores estatísticos e determinísticos como instrumento de estratificação de povoamentos clonais de Eucalyptus sp. Cerne, v.12, p.123-136, 2006.

KITAMURA, A.E.; CARVALHO, M. de P. e; LIMA, C.G. da R. Relação entre a variabilidade espacial das frações granulométricas do solo e a produtividade do feijoeiro sob plantio direto. Revista Brasileira de Ciência do Solo, v.31, p.361-369, 2007. DOI: 10.1590/S0100-06832007000200018.

LU, D.; MAUSEL, P.; BRONDÍZIO, E.; MORAN, E. Relationships between forest stand parameters and Landsat TM spectral responses in the Brazilian Amazon Basin. Forest Ecology and Management, v.198, p.149-167, 2004. DOI: 10.1016/j. foreco.2004.03.048. 
MELLO, J.M. de; DINIZ, F.S.; OLIVEIRA, A.D. de; SCOLFORO, J.R.S.; ACERBI JÚNIOR, F.W.; THIERSCH, C.R. Métodos de amostragem e geoestatística para estimativa do número de fustes e volume em plantios de Eucalyptus grandis. Floresta, v.39, p.157-166, 2009. DOI: 10.5380/rf.v39i1.13735.

MELLO, J.M. de; OLIVEIRA, M.S. de; BATISTA, J.L.F.; JUSTINIANO JÚNIOR, P.R.; KANEGAE JÚNIOR, H. Uso do estimador geoestatístico para predição volumétrica por talhão. Floresta, v.36, p.251-260, 2006. DOI: 10.5380/rf.v36i2.6454.

MENG, Q.; CIESZEWSKI, C.; MADDEN, M. Large area forest inventory using Landsat ETM+: a geostatistical approach. ISPRS Journal of Photogrammetry and Remote Sensing, v.64, p.27-36, 2009.

MENGESHA, T.; HAWKINS, M.; NIEUWENHUIS, M. The impact of plot numbers and plot configuration on the accuracy of pre-harvest stand estimates obtained by terrestrial laser scanning. Irish Forestry, v.71, p.7-26, 2014.

PALMER, D.J.; HÖCK, B.K.; KIMBERLEY, M.O.; WATT, M.S.; LOWE, D.J.; PAYN, T.W. Comparison of spatial prediction techniques for developing Pinus radiata productivity surfaces across New Zealand. Forest Ecology and Management, v.258, p.2046-2055, 2009. DOI: 10.1016/j.foreco.2009.07.057.

R CORE TEAM. R: a language and environment for statistical computing. Vienna: R Foundation for Statistical Computing, 2014. Disponível em: <http://www.R-project.org>. Acesso em: 6 out. 2014.

RIBEIRO JÚNIOR, P.J.; DIGGLE, P.J. GeoR: a package for geostatistical analysis. R-NEWS, v.1, p.15-18, 2001.

ROBERGE, C.; WULFF, S.; REESE, H.; STAHL, G. Improving the precision of sample-based forest damage inventories through two-phase sampling and post-stratification using remotely sensed auxiliary information. Environmental Monitoring and Assessment, v.188, p.213, 2016. DOI: 10.1007/s10661-016-5208-4.
ROBERTSON, G.P. GS+: geostatistics for the environmental sciences: GS+ user's guide. Plainwell: Gamma Design Software, 1998. 152p.

SINGH, T.P.; DAS, S. Predictive analysis for vegetation biomass assessment in Western Ghat region (WG) using geospatial techniques. Journal of the Indian Society of Remote Sensing, v.42, p.549-557, 2014. DOI: 10.1007/s12524-013-0335-7.

SILVA, S.T. da; MELLO, J.M. de; ACERBI JÚNIOR, F.W.; REIS, A.A. dos; RAIMUNDO, M.R.; SILVA, I.L.G.; SCOLFORO, J.R.S. Uso de imagens de sensoriamento remoto para estratificação do cerrado em inventários florestais. Pesquisa Florestal Brasileira, v.34, p.337-343, 2014. DOI: 10.4336/2014. pfb.34.80.742.

TOMPPO, E.O.; GAGLIANO, C.; DE NATELE, F.; KATILA, M.; MCROBERTS, R.E. Predicting categorical forest variables using an improved k-nearest neighbour estimator and Landsat imagery. Remote Sensing of Environment, v.113, p.500-517, 2009. DOI: 10.1016/j.rse.2008.05.021.

VIANA, H.; ARANHA, J.; LOPES, D.; COHEN, W.B. Estimation of crown biomass of Pinus pinaster stands and shrubland above-ground biomass using forest inventory data, remotely sensed imagery and spatial prediction models. Ecological Modelling, v.226, p.22-35, 2012. DOI: 10.1016/j.ecolmodel.2011.11.027.

VIEIRA, S.R. Geoestatística em estudos de variabilidade espacial do solo. In: NOVAIS, R.F.; ALVAREZ V., V.H.; SCHAEFER, C.E.G.R. (Ed.). Tópicos em Ciências do Solo. Viçosa: Sociedade Brasileira de Ciência do Solo, 2000.

WESTFALL, J.A. Strategies for the use of mixed-effects models in continuous forest inventories. Environmental Monitoring and Assessment, p.188-245, 2016. DOI: 10.1007/ s10661-016-5252-0.

YAMAMOTO, J.K.; LANDIM, P.M.B. Geoestatística: conceitos e aplicações. São Paulo: Oficina de Textos, 2013. 215p.

Recebido em 4 de fevereiro de 2016 e aprovado em 10 de junho de 2016

Pesq. agropec. bras., Brasília, v.51, n.10, p.1751-1761, out. 2016 DOI: 10.1590/S0100-204X2016001000007 